\title{
Radiación ionizante: revisión de tema y recomendaciones para la práctica
}

\author{
Aníbal E. Badel, Juan S. Rico-Mesa, María C. Gaviria, Daniela Arango-Isaza \\ y César A. Hernández Chica*
}

\author{
Cardiología intervencionista y Hemodinamia. Universidad CES, CES Cardiología, Medellín, Colombia
}

Recibido el 9 de mayo de 2017; aceptado el 23 de octubre de 2017

Disponible en Internet el 10 de abril de 2018

\section{PALABRAS CLAVE \\ Hemodinamia; Coronariografía; Intervencionismo coronario percutáneo}

\section{KEYWORDS}

Haemodynamics;

Angiography;

percutaneous

coronary

interventionism
Resumen La seguridad en radiación es un tema de actualidad. A diario se evidencian las consecuencias deletéreas de esta herramienta diagnóstica y terapéutica. En su mayoría, las patologías asociadas secundarias a la exposición por radiación podrían prevenirse con una adecuada intervención preventiva y protectora. Se presenta un artículo de revisión con el fin de explicar de forma detallada los aspectos más relevantes acerca de la formación de los rayos $X$, su definición y clasificación dosimétrica, los efectos biológicos de la radiación, los límites recomendados y las medidas de protección para la exposición de radiación.

(C) 2018 Sociedad Colombiana de Cardiología y Cirugía Cardiovascular. Publicado por Elsevier España, S.L.U. Este es un artículo Open Access bajo la licencia CC BY-NC-ND (http:// creativecommons.org/licenses/by-nc-nd/4.0/).

lonising radiation: A review of the topic and recommendations for using it

Abstract Radiation safety is a current topic. The harmful effects of this diagnostic and therapeutic tool are observed daily. The majority of associated secondary to radiation exposure could be prevented with suitable safety and protective measures. A review article is presented with the aim of explaining the most relevant aspects of radiation in detail, including the formation of $x$-rays, their dosimetry definition and classification, the biological effects of radiation, the recommended limits, as well as the protection measures for the exposure to radiation.

(C) 2018 Sociedad Colombiana de Cardiología y Cirugía Cardiovascular. Published by Elsevier España, S.L.U. This is an open access article under the CC BY-NC-ND license (http:// creativecommons.org/licenses/by-nc-nd/4.0/).

* Autor para correspondencia.

Correo electrónico: mdcessar@une.net.co (C.A. Hernández Chica). 


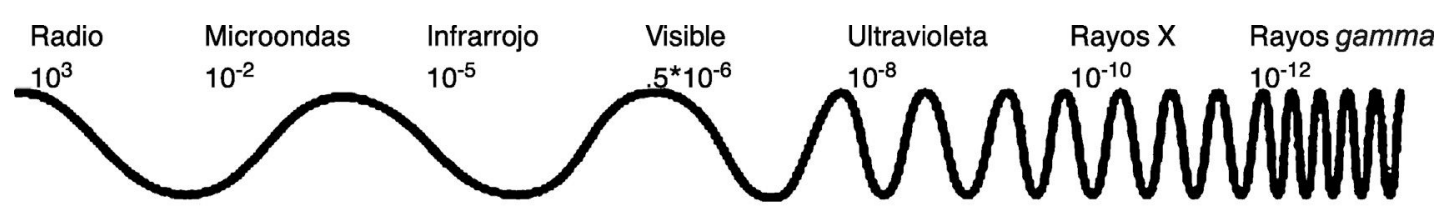

Longitud de onda en metros

Figura 1 Espectro de radiación electromagnética.

\section{Introducción}

El uso de radiación ionizante para el diagnóstico y tratamiento de ciertas patologías es la mayor fuente artificial de radiación en seres humanos. Los estudios han demostrado falta de conocimiento entre los médicos acerca de los conceptos básicos sobre este tema ${ }^{1}$.

Esta revisión busca explicar de forma simple la manera en la que se forman los rayos $X$, los efectos biológicos de la radiación, las definiciones dosimétricas de mayor importancia clínica, los límites de radiación recomendados y las medidas de protección para el paciente y el personal de salud expuesto.

\section{Metodología}

Se hizo una revisión no sistemática de la literatura apoyada en las siguientes palabras clave: hemodinamia, coronariografía, intervencionismo coronario percutáneo; en inglés y español. Los buscadores utilizados para este fin fueron: PubMed, Google scholar, Clinical Key entre otros. Se eligieron 32 referencias bibliográficas a criterio de los autores, entre ellas artículos de revisión, revisiones sistemáticas, guías terapéuticas, capítulos de libros y artículos originales de desenlaces clínicos.

\section{Conceptos básicos de formación de los rayos $\mathrm{X}$}

Un haz de rayos $X$ está formado por fotones, cada uno de los cuales contiene miles de veces la energía de un fotón de luz visible aunque menor energía que los rayos gamma. Los rayos $X$ se encuentran dentro del espectro electromagnético definido como la radiación que va desde las ondas de radio (menor cantidad de energía y mayor longitud de onda) hasta los rayos gamma (mayor energía y menor longitud de onda), como se muestra en la figura 12 .

La producción de rayos $X$ para formación de imágenes médicas ocurre en un tubo al calentar un filamento con corriente eléctrica que genera un haz de electrones. Este haz es disparado o dirigido contra un objetivo metálico pesado, que al golpear contra el núcleo de cada átomo del metal se produce una desviación de la dirección del haz de electrones, el cual sale del átomo recién ionizado (impactado) con una nueva dirección y menor energía. Adicionalmente, un porcentaje de la energía total del átomo es liberada en forma de radiación ionizante ${ }^{3}$.

Finalmente, el resultado es un haz de rayos $X$ que almacena en promedio 20 kiloelectrones voltio (KeV) de energía. El impacto de un metal pesado con un haz de electrones en un tubo de rayos $X$ se conoce como bremsstrahlung 0 radiación de choque o frenado ${ }^{4}$.

Otro proceso atómico capaz de producir rayos $X$ es la radiación tipo "K-Shell". En este caso el haz de electrones de alta energía choca contra uno de los electrones que giran en órbitas alrededor del núcleo del átomo y lo saca de la molécula; este vacío es ocupado por otro electrón que se encuentra en una órbita externa que perderá energía y se expulsará en forma de rayos $X^{3}$.

\section{Definiciones dosimétricas clínicamente importantes}

KERMA (energía cinética liberada en la materia, su sigla en inglés): energía liberada por el haz de rayos $X$ por unidad de masa de un material específico en un pequeño volumen irradiado de materia. La unidad de medida es el gray (Gy), un Gy equivale a un joule $(\mathrm{J})$ de energía por kilogramo de materia. En el caso del KERMA de los rayos X sobre los tejidos blandos esto es numéricamente equivalente a la dosis absorbida ${ }^{5}$.

Air Kerma $\left(\boldsymbol{K}_{\text {air }}\right)$ : es el Kerma en un pequeño volumen irradiado de aire. Se reporta en miligray (mGy) y caracteriza la intensidad del haz de rayos $X^{4}$.

Dosis absorbida: también llamada dosis material $\left(D_{\text {material }}\right)$ o dosis exposición. Es la energía de radiación ionizante absorbida por unidad de masa de materia irradiada en un punto específico. Su unidad del medida es el Gy. La dosis absorbida no tiene en cuenta el tipo de radiación ionizante ni la sensibilidad de expuesto. Es usual tener diferentes valores de dosis absorbida sobre el tejido irradiado durante un procedimiento y esto se debe al reposicionamiento del haz de rayos $X$, la distancia variable entre el punto focal del tubo de rayos $X$ y el tejido, así como a la variación del espesor del paciente, lo que causa cambios en el voltaje del tubo de rayos $\mathrm{X}$ y la corriente ${ }^{5}$.

Dosis equivalente: diferentes tipos de radiación, tales como rayos $X$, protones, neutrones y partículas alfa causan diversos grados de daño biológico por unidad de dosis absorbida. Teniendo en cuenta esta diferencia, se puede utilizar un factor de ponderación basado en el tipo de radiación utilizada. Por definición, el factor de ponderación de los rayos $X$ es 1. La unidad de medida es el sievert $(\mathrm{Sv})^{5}$.

Dosis efectiva $(E)$ : los tejidos tienen grados variables de radiosensibilidad. Por ejemplo, mama, médula ósea y colon son más radiosensibles que la superficie ósea, el cerebro y la piel. $\boldsymbol{E}$ es la suma promedio de la radiación recibida de todos los tejidos expuestos y tiene en cuenta para su cálculo la radiosensibilidad de cada tejido. Su valor se obtiene 
Tabla 1 Dosis promedio efectiva de radiación ionizante en procedimientos endovasculares, equivalente en número de radiografías de tórax y años de exposición a radiación natural ${ }^{6,31}$

\begin{tabular}{llll}
\hline Procedimiento & Dosis efectiva en mSv & $\begin{array}{l}\text { Número equivalente } \\
\text { en radiografías de } \\
\text { tórax (cada 0,02 mSv) }\end{array}$ & $\begin{array}{l}\text { Equivalente promedio } \\
\text { de radiación natural } \\
\text { (en años) }\end{array}$ \\
\hline Angiografía coronaria diagnóstica & & 350 & 2.9 \\
Intervención coronaria percutánea & $2-16$ & 750 & 6.3 \\
Ablación por radiofrecuencia & $7-57$ & 750 & 6.3 \\
Angiografía de cabeza y/o cuello & $7-57$ & 250 & 2.1 \\
Angiografía torácica de arteria pulmonar o aorta & $4-90$ & 250 & 2.1 \\
Angiografía abdominal & $4-48$ & 600 & 5.0 \\
Embolización de vena pélvica & $44-78$ & 3000 & 25.0 \\
Valvuloplastia aórtica & 39 & 1950 & 16.2 \\
Dilatación de oclusión coronaria crónica & $17-194$ & 4050 & 33.7 \\
Endoprótesis de aneurisma toraco-abdominal & $76-119$ & $3800-5950$ & $31.6-49.5$ \\
\hline
\end{tabular}

al multiplicar la dosis absorbida por un valor de ponderación (n) previamente definido para cada tejido expuesto. Se expresa en milisieverts (mSv) y es útil para comparar la cantidad de radiación recibida por diferentes tipos de procedimientos, pero no es una medida útil de riesgo individual de radiación del paciente expuesto (tabla 1) 1,5,6.

Dosis de radiación a la entrada de la piel (entrance skin dose, ESD): es la dosis de radiación absorbida por la piel. Este valor generalmente no se puede informar con exactitud pero puede estimarse si se conoce el aire KERMA de entrada (entrance air KERMA, EAK) a nivel de la piel. El EAK se multiplica por un factor que tiene en cuenta las ligeras diferencias en la energía absorbida por el aire y los tejidos blandos. Al entrar en contacto del haz de rayos $X$ con el paciente se genera parte de la radiación dispersa y esto aumenta el valor medido de ESD ${ }^{1,5}$.

Dosis pico en la piel (peak skin dose, PSD): es la máxima ESD en la zona de la piel más fuertemente irradiada. Esto corresponde a la zona que esta más tiempo durante el procedimiento en contacto con la columna primaria del haz de rayos $X$. Esta medida es dispendiosa para realizar en la práctica diaria ${ }^{1,5}$.

Dosis umbral: es la mínima dosis a la cual se pueden presentar reacciones tisulares. Varía entre los individuos y los tipos de tejido ${ }^{5}$.

Radiación dispersa: es la que se genera por el cuerpo del paciente y es la principal fuente de exposición del personal de la sala. ${ }^{5}$

Tiempo de fluoroscopia: es el período de tiempo que se utiliza la fluoroscopia durante un procedimiento. Es la medida menos útil para estimar dosis o riesgo, puesto que, no tiene en cuenta la intensidad del haz, el número de fotos por segundo, la colimación, el tipo de fluoroscopia, entre otras $^{5}$.

KERMA producto de área, (KAP o DAP): se considera la mejor forma de medir el riesgo estocástico para el paciente y se expresa en $\mathrm{Gy} / \mathrm{cm}^{2}$; no indica la probabilidad de reacción en piel. Mide principalmente la cantidad de radiación recibida por área transversal de tejido expuesto del paciente pero también la radiación dispersa que recibe el operador y el personal de la sala. Su valor se calcula al multiplicar la intensidad del haz de rayos $\mathrm{X}$ o air KERMA $\left(\mathrm{K}_{\mathrm{air}}\right)$ por el área de sección transversal de tejido expuesto, así: KAP Gy/ $\mathrm{cm}^{2}$ : $\left(\mathrm{K}_{\mathrm{air}} \mathrm{Gy}\right) \mathrm{x}$ (área transversal $\mathrm{cm}^{2}$ ); este valor debe ser reportado en la historia clínica del paciente que sea expuesto a un procedimiento que utilice rayos $X^{7}$.

Punto de referencia air KERMA $\left(K_{a, r}\right)$ : es la dosis total acumulada de $\mathbf{K}_{\mathrm{air}}$ en un punto de referencia internacionalmente definido durante la realización de un procedimiento y se expresa en Gy. La localización de este punto a $15 \mathrm{~cm}$ del isocentro hacia el tubo de rayos $X$ varía en los diferentes equipos de fluoroscopia (encima, a nivel o por debajo de la piel) y es la mejor forma de medir la lesión en la piel por radiación. El $K_{\text {air }}$ no tiene en cuenta el movimiento del arco $y$, por tanto, el valor de radiación medida se da como si fuera en un punto fijo; esto hace que la medición de radiación de piel no sea completamente precisa y tienda a sobreestimar la medición ${ }^{8}$.

\section{Efectos biológicos de la radiación}

A diferencia de la luz visible, los rayos $X$ tienen la suficiente energía para penetrar los tejidos humanos y ocasionar reacciones ionizantes, lo que trae consigo potencial mutagénico ${ }^{9}$. Todos los seres humanos están expuestos a radiación proveniente de fuente natural o artificial (fig. 2). La dosis anual promedio de radiación recibida por una persona es alrededor 2-3 mSv de E/año mientras que la creada por fuentes médicas es más de la mitad de este valor ${ }^{10}$.

Los efectos biológicos deletéreos que produce la radiación ionizante se explican por dos razones: efecto estocástico y reacciones tisulares (anteriormente denominado efecto determinístico). Estocástico es la probabilidad de daño no reparado del $A D N$, lo que se traduce en cáncer radiogénico (CR) (generalmente neoplasias hematológicas) ${ }^{5}$. El tiempo entre el evento índice y la aparición de la enfermedad clínicamente manifiesta es variable y puede tardar hasta 20 años en manifestarse ${ }^{4}$. El riesgo de cáncer aumenta mientras mayor sea la exposición, sin embargo, la gravedad del CR es independiente de la dosis de radiación. Así mismo, la incidencia es mayor en mujeres que en hombres ${ }^{9,11}$.

Cabe anotar que cuanto más joven mayor es el incremento del riesgo para padecer un $C R$ en ambos géneros, 


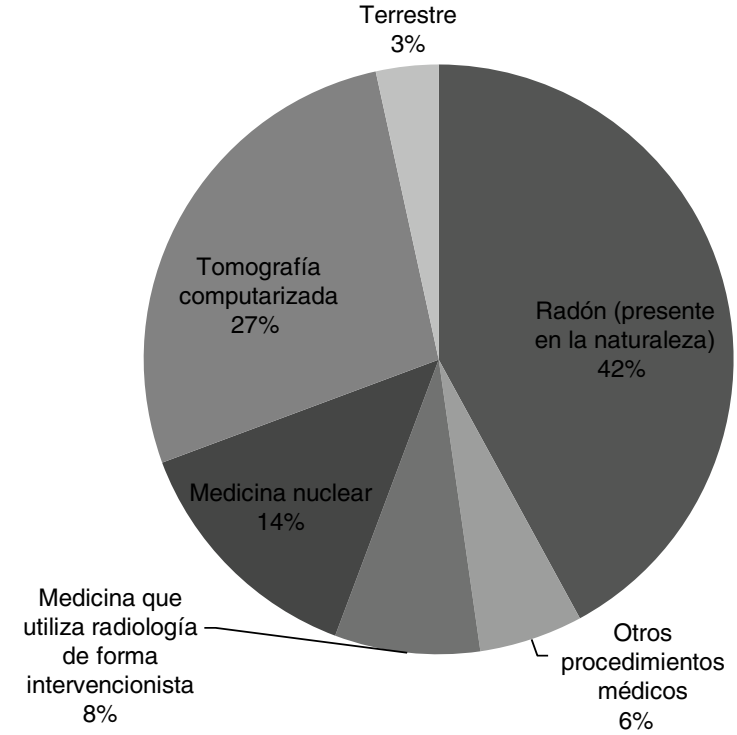

Figura 2 Representación porcentual de fuentes de radiación ionizante naturales y artificiales ${ }^{9,10}$.

independiente de la fuente de radiación. Entre las mujeres la fuente de radiación que más incrementa el riesgo de cáncer es la angiografía coronaria por tomografía computarizada (CCTA, su sigla en inglés) sin técnicas adecuadas de optimización, seguido de CCTA con adecuadas técnicas de optimización. En los hombres la fuente de mayor riesgo de cáncer es la imagen de perfusión miocárdica realizada por tomografía computarizada de emisión de fotones individuales (SPECT MPI, su sigla en inglés) seguido del CCTA sin técnicas adecuadas de optimización. El riesgo máximo en las mujeres es 0,47\% aproximadamente, mientras que en los hombres es de $0,27 \%^{12-14}$. En cuanto a los procedimientos de Cardiología intervencionistas más frecuentes y la dosis efectiva de exposición a radiación ionizante de los pacientes ver la tabla 1 en donde se comparan con su equivalente en $\mathrm{Rx}$ de tórax y a años de exposición a radiación natural.

Según las directrices del Consejo Nacional de Protección Radiológica (ICRP) una ocupación con menos de 1 muerte por cada 10.000 trabajadores/año se considera segura. De los especialistas que trabajan con radiación ionizante, los cardiólogos intervencionistas son lo que tienen mayor exposición anual a radiación (fig. 3) ${ }^{15}$.

Un cardiólogo intervencionista tiene una exposición anual a radiación que oscila entre 2-5 mSv año (equivalente a 150 radiografías de tórax año); en 30 años de trabajo la dosis de radiación aproximada equivale a $100 \mathrm{mSv}$. Dicha cantidad de radiación acumulada se asocia con la aparición de cáncer (mortal y no mortal) por cada 100 sujetos expuestos y un cáncer mortal en 200 sujetos expuestos ${ }^{16}$.

Hay dos efectos estocásticos que son importante mencionar. Uno es el reporte de mayor incidencia de tumores en el lado izquierdo del cerebro en cardiólogos y radiólogos intervencionistas; sin embargo, no se ha podido demostrar de manera consistente una relación directa entre la radiación craneal y la aparición de neoplasias cerebrales como glioblastoma multiforme, así como tampoco mayor incidencia de alteraciones del comportamiento como depresión o ansiedad (dosis crónicas bajas de radiación pueden

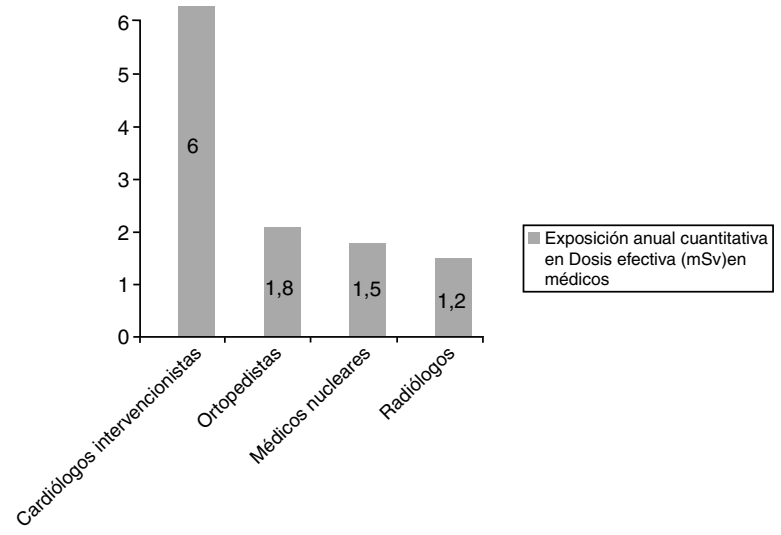

Figura 3 Exposición anual a radiación para las diferentes especialidades médicas ${ }^{15}$.

causar efecto deletéreo en la neurogénesis del hipocampo y la plasticidad neuronal). Probablemente, esto no haya sido demostrado porque se requieren estudios con mayor cantidad de pacientes y mejor diseño para evitar factores confusores $^{17}$.

El segundo efecto es la mayor incidencia de opacidades subcapsulares posteriores del cristalino, que han sido documentadas hasta en un $50 \%$ de los cardiólogos intervencionistas, y cataratas. Las razones de esta alta prevalencia se explican por la exposición del operador a la radiación dispersa, la poca utilización de las gafas de plomo $(<$ del $30 \%$ de los operadores) y los límites previos de dosis ocupacional legales, que fueron demasiado altos para tener preocupación de este efecto ${ }^{18-21}$.

Se recomienda la utilización de cubiertas plomadas para la cabeza (gorros plomados), lentes plomados (disminuyen la dosis de radiación entre un 35 a 95\% según diferentes estudios y diseños de lentes) y escudos (vidrios plomados 0 mamparas) entre el paciente y el operador, para disminuir la dosis de radiación craneal y facial recibida ${ }^{17}$.

Las reacciones tisulares son alteraciones producidas en los tejidos humanos por desnaturalización de proteínas secundario a exposición a radiación ionizante y dependiente de dosis. Según la intensidad de la dosis de radiación recibida, tiempo de exposición y sensibilidad del tejido las lesiones pueden aparecer en horas, días, semanas o meses y comportarse como transitorias o permanentes ${ }^{22}$.

La piel es el tejido más afectado por ser la primera barrera que recibe el haz de rayos $X$. Las lesiones pueden ir desde eritema transitorio, depilación dérmica y caída del cabello, hasta úlceras profundas permanentes (tabla 2). Pacientes sometidos a procedimientos que superen una dosis de radiación de $5.000 \mathrm{mGy}$ de $\mathrm{K}_{\mathrm{a}, \mathrm{r}}$ deben tener seguimiento por el riesgo de lesión de la piel ${ }^{23,24}$.

El estado de salud del paciente puede modificar la respuesta normal de la piel o de otros órganos a la radiación; enfermedades del colágeno, diabetes mellitus, hipertiroidismo favorecen la generación de lesiones ${ }^{25}$. Por tanto, utilizar la mínima dosis de radiación posible para evitar alcanzar dosis umbrales y el estrecho seguimiento de los pacientes que lo requieran, son medidas fundamentales para prevenir y manejar la aparición de lesiones en piel. 
Tabla 2 Tipo de lesión celular inducida por radiación durante procedimientos guiados por fluoroscopia ${ }^{19,20}$

\begin{tabular}{lllll}
\hline Efecto de la radiación & $\begin{array}{l}\text { Límite de la dosis } \\
\text { PSD (Gy) }\end{array}$ & $\begin{array}{l}\text { Tiempo de } \\
\text { fluoroscopia baja } \\
(20 \mathrm{mGy} / \mathrm{min})\end{array}$ & $\begin{array}{l}\text { Tiempo de } \\
\text { fluoroscopia alta } \\
(200 \mathrm{mGy} / \mathrm{min})\end{array}$ & $\begin{array}{l}\text { Tiempo en que } \\
\text { aparece el efecto }\end{array}$ \\
\hline Eritema transitorio temprano & 2 & $1 \mathrm{~h} 42 \mathrm{~min}$ & $10 \mathrm{~min}$ & horas \\
Depilación temporal & 3 & $2 \mathrm{~h} 30 \mathrm{~min}$ & $15 \mathrm{~min}$ & 3 semanas \\
Eritema principal & 6 & $5 \mathrm{~h}$ & $30 \mathrm{~min}$ & 10 días \\
Depilación permanente & 7 & $6 \mathrm{~h}$ & $35 \mathrm{~min}$ & 3 semanas \\
Descamación seca & 10 & $8 \mathrm{~h}$ & $50 \mathrm{~min}$ & 4 semanas \\
Atrofia dérmica & 11 & $9 \mathrm{~h}$ & $55 \mathrm{~min}$ & Más de 14 semanas \\
Telangiectasia & 12 & $10 \mathrm{~h}$ & $1 \mathrm{~h}$ & Más de 52 semanas \\
Descamación húmeda & 15 & $12 \mathrm{~h}$ & $1 \mathrm{~h} 15 \mathrm{~min}$ & 4 semanas \\
Eritema tardío & 15 & $12 \mathrm{~h}$ & $1 \mathrm{~h} 15 \mathrm{~min}$ & $6-10$ semanas \\
Necrosis dérmica & 18 & $15 \mathrm{~h}$ & $1 \mathrm{~h} 30 \mathrm{~min}$ & $1 \mathrm{~h} 40$ min \\
Ulceración secundaria & 20 & $17 \mathrm{~h}$ & & Más de 10 semanas \\
\hline
\end{tabular}

Tabla 3 Medidas dosimétricas de radiación fluoroscópica

\begin{tabular}{|c|c|c|c|}
\hline Medida dosimétrica & Unidad & $\begin{array}{l}\text { Utilidad clínica de la medición } \\
\text { dosimétrica }\end{array}$ & $\begin{array}{l}\text { Nivel dosimétrico } \\
\text { limite }^{*}\end{array}$ \\
\hline $\begin{array}{l}\text { Aire total KERMA en el } \\
\text { punto de referencia }\left(\mathrm{K}_{\mathrm{a}, \mathrm{r}}\right)\end{array}$ & Gy & $\begin{array}{l}\text { Predictor del riesgo de daño cutáneo; } \\
\mathrm{K}_{\mathrm{a}, \mathrm{r}} \text { no es una medida directa de dosis } \\
\text { máxima en piel }\end{array}$ & $5 \mathrm{~Gy}$ \\
\hline $\begin{array}{l}\text { Producto de Aire KERMA - } \\
\text { Área }\left(\mathrm{P}_{\mathrm{ka}}\right) \\
\text { (también conocido como } \\
\text { producto dosis-área) }\end{array}$ & $\mathrm{Gy}-\mathrm{cm}^{2}$ & $\begin{array}{l}\text { Valor integrado del Aire KERMA } \\
\text { entregado al paciente, usado para } \\
\text { calcular el riesgo relativo } \\
\text { estocástico; No un riesgo absoluto } \\
\text { medido } \\
\text { Altos niveles de } \mathrm{P}_{\mathrm{ka}} \text {, cuando } \mathrm{K}_{\mathrm{a}, \mathrm{r}} \text {, es } \\
\text { constante, implica que más de un } \\
\text { tejido fue irritado. }\end{array}$ & $\begin{array}{l}500 \mathrm{~Gy} \mathrm{~cm}^{2} \text { para } \\
\text { un campo de } 100 \\
\mathrm{~cm}^{2} \text { en la piel }\end{array}$ \\
\hline Tiempo de fluoroscopia & Minuto & $\begin{array}{l}\text { Utilidad mínima debido a que no es } \\
\text { afectada por la talla del paciente, } \\
\text { ángulo del rayo, cineangiografía u } \\
\text { otros factores de relevancia }\end{array}$ & 60 minutos \\
\hline
\end{tabular}

\section{Límites de radiación recomendados}

Por regulación de la FDA, todos los equipos de fluoroscopia fabricados a partir de 2006 deben tener la capacidad de mostrar el valor de KAP, $\mathrm{K}_{\mathrm{a}, \mathrm{r}} \mathrm{y}$ el tiempo de fluoroscopia ${ }^{26,27}$. Estas medidas dosimétricas deben ser reportadas en la historia clínica del paciente.

Existen también recomendaciones y límites de radiación para el operador y personal de la sala (tabla 4): utilización simultánea de dos dosímetros, uno por debajo del delantal el cual da una estimación razonable de la dosis efectiva en la mayoría de los casos y otro por fuera del delantal en el cuello (lado izquierdo), que da una estimación de la radiación recibida en cabeza y ojos ${ }^{18}$. Esta recomendación no se lleva a cabo con frecuencia en la práctica diaria, en parte por el temor del especialista a ser retirado temporalmente de la sala en caso de exceder el límite de radiación y en otras oportunidades porque no están convencidos de su valor ${ }^{28}$.
Tabla 4 Recomendaciones y límites de radiación para el operador y personal de la sala

Límite dosimétrico para exposición ocupacional

\begin{tabular}{ll}
\hline Cantidad de dosis & Límite de dosis ocupacional \\
Dosis efectiva & $\begin{array}{l}20 \mathrm{mSv} \text { por año promediado } \\
\text { por cinco años consecutivos } \\
\text { (100 mSv en } 5 \text { años), y } 50 \mathrm{mSv} \\
\text { en algún año individual }\end{array}$ \\
$\begin{array}{ll}\text { Dosis equivalente en: } & \text { por períodos definidos de cinco } \\
\text { Lentes del ojo } & \text { años, sin años individuales sin } \\
& \text { exceder } 50 \mathrm{mSv}\end{array}$ \\
$\begin{array}{l}500 \mathrm{mSv} \text { en un año } \\
\text { Piel }\end{array}$ & $500 \mathrm{mSv}$ en un año \\
\hline
\end{tabular}

* Promediado por $1 \mathrm{~cm}^{2}$ de las áreas de piel más irradiadas. Modificada de las recomendaciones del ICRP. 


\section{Medidas para disminuir la dosis de radiación}

Todo procedimiento médico que implique el uso de radiación debe estar debidamente justificado. Una vez definida la necesidad de exposición, se deben optimizar al máximo las medidas de radioprotección de acuerdo con el principio ALARA (del inglés as low as reasonably achievable) que traduce: "tan bajo como sea razonablemente posible" 29 .

La norma de la comisión electrotécnica 60601-2-43 estipula que el equipo de rayos $X$ intervencionista proporcione un informe de la estructura de la dosis de radiación (RDSR) y se disponga de un listado completo de dispositivos y accesorios de protección ${ }^{17}$.

El especialista debe conocer la $E$ promedio esperada para cada procedimiento reportada en la literatura (niveles de referencia diagnósticos, $\mathrm{DRL}_{\mathrm{s}}$ ) (tabla 1) para así poder comparar con lo que está haciendo en su sitio de trabajo; se debe seleccionar en el equipo de fluoroscopia el programa adecuado para cada procedimiento. Otra estrategia recomendada es trabajar con la menor dosis de fluoroscopia posible que permita una calidad de imagen suficiente para una toma de decisiones diagnósticas o terapéuticas adecuada. Las imágenes pueden ser obtenidas con una modalidad de fluoroscopia alta (mayor cantidad de radiación a expensas de mayor calidad de imagen), media baja (menor cantidad de radiación a expensas de menor calidad en la imagen) ${ }^{1}$.

Hay dos modalidades de adquisición de imágenes: cine y sustracción digital (DSA). La modalidad de adquisición de angiografía en sustracción digital (DSA) toma una primera imagen del árbol vascular previo a la inyección del contraste, lo que ofrece una perspectiva de toda la estructura orgánica; esta se almacena en la memoria de la computadora, después se inyecta el contraste y se hace una segunda imagen que refleja el flujo sanguíneo. Aesta segunda toma se le restan las imágenes que no corresponden al árbol vascular con lo que se logra una angiografía final de los vasos sanguíneos en estudio. Con esta técnica se llega a tener una resolución tal que se pueden ver vasos de un milímetro de diámetro que con fluoroscopia o cine difícilmente se podrían visualizar, esto a expensas de una mayor cantidad de radiación. Dicho de una forma más sencilla, la dosis de radiación recibida con un minuto de cine es la equivalente promedio a 10 minutos de fluoroscopia y, un minuto de DSA es equivalente a 10 minutos de cine ${ }^{30}$. La recomendación es tratar de utilizar la DSA sólo cuando la situación lo amerite.

La posición de tubo de rayos $X$ respecto al paciente y la altura de la mesa son otros de los factores a tener en cuenta para disminuir la radiación, así: colocar la mesa a la altura adecuada para que el operador este cómodo, aumentar la distancia entre el tubo de rayos $X$ y el paciente (al menos $50 \mathrm{~cm}$ ), disminuir la distancia entre el intensificador de imagen y el paciente para evitar en lo posible las proyecciones oblicuas, sobre todo las oblicuas izquierdas, así como las laterales izquierdas (los rayos $\mathrm{X}$ son dirigidos hacia el operador) que aumentan la dosis de radiación recibida por el operador, puesto que generan más radiación dispersa.

Colimar es orientar el haz de rayos $X$ con la ayuda de unos filtros dentro del tubo de rayos $X$ al campo de visión de trabajo. Con esto se reduce tanto la radiación recibida por el paciente como la radiación dispersa. En consecuencia, la

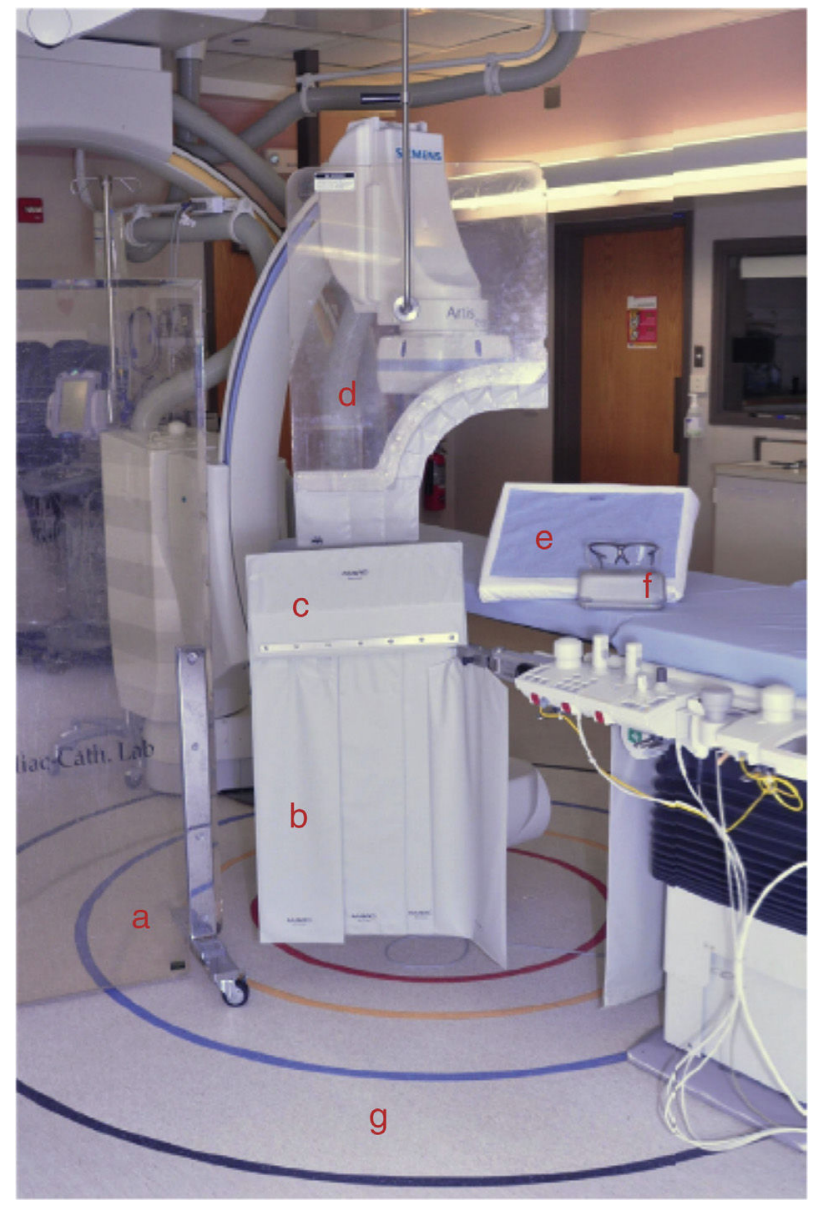

Figura 4 Dispositivos para la protección de la radiación: a)Escudo plomado de vidrio protector.; b) Escudo plomado protector de miembros inferiores; c) Escudo con extensión protectora vertical; d) escudo plomado de protección toracoabdominal; e) Dispositivo para la absorción ionizante, de carácter desechable y estéril; f) Gafas con protección radioionizante; (g) Las líneas en el suelo representan la distancia desde el paciente a la que el límite al cual puede acceder el profesional de la salud.

colimación minimiza la dosis de radiación absorbida por el paciente, así como la dosis de radiación dispersa, y mejora la calidad de la imagen ${ }^{13}$.

No utilizar en lo posible magnificación de la imagen a menos que sea necesario. No dar rayos cuando no se esté mirando la pantalla. El segundo operador, en la medida de las posibilidades, se debe ubicar detrás y al lado del primer operador para que la mayoría de la radiación dispersa sea bloqueada por el primer operador ${ }^{27}$.

Respecto a los dispositivos de blindaje (fig. 4), se refieren a la colocación de cualquier dispositivo entre la máquina que genera la radiación ionizante y las personas (paciente y equipo humano) con el propósito de disminuir la cantidad de radiación dispersa que llega al personal de la sala. Los implementos que debe vestir el personal presente en la sala, diferente al paciente son: gorro plomado, gafas con protección radioionizante, collar plomado de protección radioionizante para evitar el efecto sobre la tiroides, 


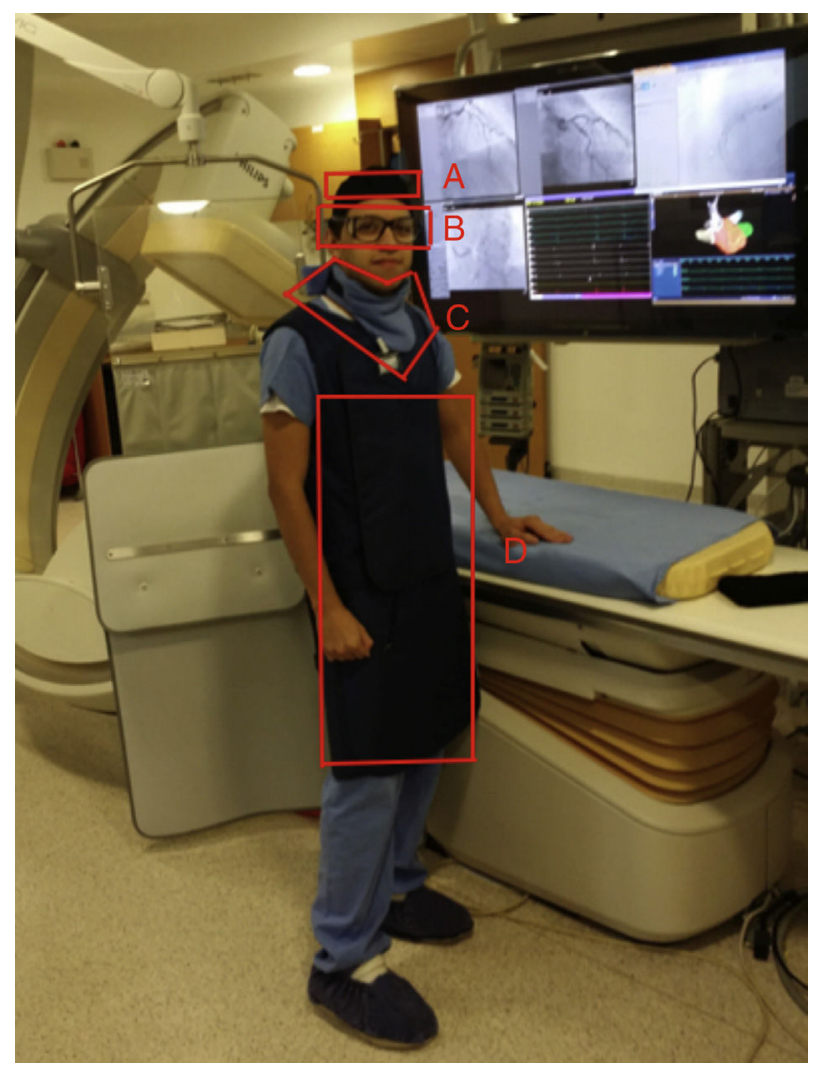

Figura 5 Medidas de protección vascular personal. A: Gorro plomado, B: Gafas con protección radioionizante, C: Collar plomado de protección radio- ionizante, $\mathrm{D}$ : Chaleco plomado para protección de miembros inferiores, abdomen y tórax.

chaleco plomado de $0,5 \mathrm{~mm}$ de plomo o su equivalente, idealmente de 2 piezas (70\% en la cadera y $30 \%$ en los hombros), y en lo posible pantorrilleras y hombrera izquierda (fig. 5$)^{29}$.

En cuanto a la protección del paciente, este se beneficia de las medidas que se usan para la protección de la sala y el personal que realiza el procedimiento, siempre bajo la premisa del principio ALARA y fundamentado en que el beneficio que recibirá el paciente supera claramente el riesgo por la radiación ionizante (adecuada indicación del procedimiento). En la protección del pacientes (v.g. embarazadas) se pueden usar chalecos plomados para disminuir el área de exposición (el abdomen en la embarazada) y en algunos casos campos estériles plomados que a su vez disminuyen la radiación dispersa.

\section{Conclusiones}

Las intervenciones diagnósticas y terapéuticas que requieren equipos que emiten radiación ionizante son, hasta la fecha, necesarios en muchos campos de la Medicina. Los cardiólogos intervencionistas son el grupo de profesionales que mayor radiación ionizante reciben. Por tanto, es imperativo contar con excelentes medidas de radioprotección para el paciente y el personal de salud que trabaja con estos equipos.

\section{Financiación}

Ninguna.

\section{Conflicto de intereses}

Ninguno.

\section{Bibliografía}

1. Moscucci M. Cineangiography imaging, radiation safety and contrast agents. In: Grossman \& Baim's Cardiac Catheterization, Angiography, and Intervention. Eighth Edition. Lippincott Williams \& Wilkins;.

2. Textbook of Interventional Cardiology - 9780323340380 | US Elsevier Health Bookshop [Internet]. [Acceso 8 May 2017]. Disponible en: https://www.us.elsevierhealth.com/textbook-ofinterventional-cardiology-9780323340380.html

3. Bushberg J, Siebert J, Leidthholdt E. The essential physics of medical imaging. In: Second edition. Lippincott, Williams \& Wilkins.

4. Eric J. Radiation Safety During Cardiovascular Procedures. In: Textbook of interventional cardiology. Seventh edition.

5. Dixon R, Ogden K. A field guide to radiation safety terminology. [Acceso 8 May 2017]. Disponible en: http://evtoday.com/ pdfs/et0816_F1_Dixon.pdf

6. Mettler FJ, et al. Effective doses in radiology and diagnostic nuclear medicine: a catalog. - PubMed - NCBI. [Acceso 24 Jul 2016]. Disponible en: http://www.ncbi.nlm.nih.gov/ pubmed/18566177

7. Venneri L, et al. Cancer risk from professional exposure in staff working in cardiac catheterization laboratory: insights from the National Research Council's Biolog. - PubMed - NCBI. [Acceso 24 Jul 2016]. Disponible en: http://www.ncbi.nlm. nih.gov/pubmed/19081407

8. Vano $\mathrm{E}$, et al. Radiation cataract risk in interventional cardiology personnel. - PubMed - NCBI. [Acceso 24 Jul 2016]. Disponible en: http://www.ncbi.nlm.nih.gov/pubmed/? term $=30 . \% 09$ Vano $+E \% 2 C+$ Kleiman $+N J \% 2 C+$ Duran $+A \% 2 C+$ Rehani+ $\mathrm{MM} \% 2 \mathrm{C}+$ Echeverri+D\%2C+Cabrera+M.+Radiation+cataract+ risk+in+interventional+cardiology+personnel.+Radiat+Res.+ 2010\%3B174\%3A490\%E2\%80\%93495.

9. Eugenio P, Andreassi MG, Rehani MM. Radiation protection. In: The PCR-EAPCI Textbook - Percutaneous interventional cardiovascular medicine.

10. Mettler F, Upton A. Medical effects of ionizing radiation. Third edition Philadelphia: Saunders/Elsevier; 2008.

11. Hall E, Giaccia A. Radiobiology for the Radiologist. Seventh edition Philadelphia: Wolters Kluwer Health/Lippincott Williams \& Wilkins; 2012.

12. Fazel $\mathrm{R}$, et al. Approaches to enhancing radiation safety in cardiovascular imaging: a scientific statement from the American Heart Association. - PubMed - NCBI. [Acceso 24 Jul 2016]. Disponible en:http://www.ncbi.nlm.nih.gov/pubmed/? term $=31 . \% 09$ Approaches+to+Enhancing+Radiation+Safety+in+ Cardiovascular+Imaging\%3A+A+Scientific+Statement+From+ the+American+Heart+Association.+Circulation.+ 2014\%3B130\%3A1730-1748.

13. Radiation Risk in Perspective - radiationrisk.pdf [Internet]. [Acceso 8 May 2017]. Disponible en: https://hps.org/ documents/radiationrisk.pdf

14. Einstein A, et al. Estimating risk of cancer associated with radiation exposure from 64-slice computed tomography coronary angiography. - PubMed - NCBI. [Acceso Jul 24 2016]. Disponible en: http://www.ncbi.nlm.nih.gov/pubmed/17635892 
15. Panuccio G. Comparison of indirect radiation dose estimates with directly measured radiation dose for patients and operators during complex endovascular proced. - PubMed NCBI. [Acceso Jul 24 2016]. Disponible en: http://www.ncbi. nlm.nih.gov/pubmed/21292431

16. Vañó E. et al. Radiation exposure to medical staff in interventional and cardiac radiology. - PubMed - NCBI. [Acceso Jul 24 2016]. Disponible en: http://www.ncbi.nlm. nih.gov/pubmed/10195011

17. Bartal G, Vañó E, Paulo G, Roguin A. Endovascular Today Minimizing Radiation Risk to Patients and Staff [Internet]. Endovascular Today. [Acceso 24 Feb 2017]. Disponible en: http: / /evtoday.com/2016/08/minimizing-radiation-risk-topatients-and-staff/

18. Balter $S$, et al. Fluoroscopically guided interventional procedures: a review of radiation effects on patients' skin and hair. - PubMed - NCBI. [Acceso 24 2016]. Disponible en: http: / / www.ncbi.nlm.nih.gov/pubmed/?term=Balter+S\%2C +Hopewell+JW\%2C+Miller+DL\%2C+et+al\%3A+Fluoroscopically+ guided+interventional+procedures $\% 3 \mathrm{~A}+\mathrm{a}+$ review+of+ radiation+effects+on+patients\%E2\%80\%99+skin+and+hair.+ Radiology+254\%5B2\%5D\%3A326-341\%2C+2010

19. Padovani R, Le Heron J, Cruz-Suárez R, Duran A, Lefaure C, Miller DL, et al. International project on individual monitoring and radiation exposure levels in interventional cardiology. Radiat Prot Dosimetry. 2011;144(1-4):437-41.

20. Rehani MM, Vano E, Ciraj-Bjelac O, Kleiman NJ. Radiation and cataract. Radiat Prot Dosimetry. 2011;147(1-2):300-4.

21. Huang B, Li J, Law MW-M, Zhang J, Shen Y, Khong PL. Radiation dose and cancer risk in retrospectively and prospectively ECGgated coronary angiography using 64-slice multidetector $\mathrm{CT}$. Br J Radiol. 2010;83:152-8.

22. HB, et al. Radiation dose and cancer risk in retrospectively and prospectively ECG-gated coronary angiography using 64-slice multidetector CT. - PubMed - NCBI. [Acceso Jul 24 2016]. Disponible en: http://www.ncbi.nlm.nih.gov/pubmed/20139263

23. Hirshfeld et al. JJ. ACCF/AHA/HRS/SCAl clinical competence statement on physician knowledge to optimize patient safety and image quality in fluoroscopically guided inva. - PubMed -
NCBI. [Acceso Jul 24 2016]. Disponible en: http://www.ncbi. nlm.nih.gov/pubmed/15687141

24. Mettler FJ, et al. Radiation injuries after fluoroscopic procedures. - PubMed - NCBI. [Acceso Jul 24 2016]. Disponible en: http://www.ncbi.nlm.nih.gov/pubmed/12509113

25. Balter S, Hopewell JW, Miller DL, Wagner LK, Zelefsky MJ. Fluoroscopically guided interventional procedures: a review of radiation effects on patients' skin and hair. Radiology. 2010;254:326-41.

26. Balter S, Miller DL. The new Joint Commission sentinel event pertaining to prolonged fluoroscopy. J Am Coll Radiol JACR. 2007; 4:497-500.

27. Report No. 168 - Radiation Dose Management for Fluoroscopically-Guided Interventional Medical Procedures (2010) [Internet]. [Acceso Jul 24 2016]. Disponible en: http: / / www.ncrppublications.org/Reports/168

28. Cindy. NCRP Reports 122| Bethesda, MD [Internet]. NCRP | Bethesda, MD. [Acceso Jul 24 2016]. Disponible en: http://ncrponline.org/publications/reports/ncrp-reports-122/

29. Roguin A., Bartal G. Radiation and your brain. [AcCceso 8 May 2017]. Disponible en: http://evtoday.com/ pdfs/et0816_F6_Roguin.pdf

30. Lanzer $P$, editor. Catheter-Based Cardiovascular Interventions [Internet]. Berlin, Heidelberg: Springer Berlin Heidelberg; 2013 [Acceso 8 May 2017]. Disponible en: http://link.springer.com/ 10.1007/978-3-642-27676-7

31. Suzuki S, et al. Patients' skin dose during percutaneous coronary intervention for chronic total occlusion. - PubMed NCBI. [Acceso Jul 24 2016]. Disponible en: http://www.ncbi. nlm.nih.gov/pubmed/17932885

32. Padovani R, et al. International project on individual monitoring and radiation exposure levels in interventional cardiology. - PubMed - NCBI. [Acceso Jul 24 2016]. Disponible en: http: / / www.ncbi.nlm.nih.gov/pubmed/?term=28.+Padovani+ $\mathrm{R} \% 2 \mathrm{C}+$ Le + Heron $+\mathrm{J} \% 2 \mathrm{C}+$ Cruz-Suarez+R\%2C+Duran+A\%2C+ Lefaure+C\%2C+Miller+DL\%2C+Sim+HK\%2C+Vano+E\%2C+Rehani+ $\mathrm{M} \% 2 \mathrm{C}+\mathrm{Czarwinski+R} .+$ International+project+on+individual+ monitoring+and+radiation+exposure+levels+in+interventional+ cardiology 